\title{
EDITORIAL
}

\section{Filling the gap: international reference values for health care in children}

International Journal of Obesity (2014) 38, S2-S3; doi:10.1038/ ijo.2014.129

Paediatricians often face the problem that reference values for routine clinical parameters are not available or are of questionable value for the assessment of a given child's health status, especially if the child is young. Many physiological parameters undergo important changes as children grow up, and the range of normal values changes accordingly. This supplement uses a uniquely valuable data source to address this problem, and offers reference standards for both clinical markers and key health-related behaviours or characteristics. The background data are drawn from the IDEFICS study, which surveyed more than 18000 2-11-year-old children from eight European countries on the basis of a highly standardised protocol in the years 2007-2008 and 2009-2010. ${ }^{1}$ Following pre-test of all survey modules, field personnel from each study centre participated in central training as well as further local training sessions. The coordinating centre conducted site visits to each study location during field surveys to check adherence to the standard operating procedures. All study centres used the same technical equipment, which was purchased centrally to maximise the comparability of data. Databases and computer-assisted questionnaires included automated plausibility checks. All numerical variables were entered twice independently.

Measurements included several anthropometric characteristics, a variety of metabolic blood parameters, blood pressure, ultrasonography of the heel bone, objectively measured physical activity and parental questionnaires on their child's dietary behaviour. The parameters were selected in order to investigate diet- and lifestyle-related diseases in children, with particular focus on overweight, obesity and related disorders. This rich data source offers a unique opportunity to describe the age- and sexspecific distribution of physiological parameters that are of clinical relevance, as well as of health-related states and behaviours in a diverse European population of unselected healthy children. It may also serve as a reference for the daily clinical practice of paediatricians and other health professionals.

Part 1 of this supplement provides age- and sex-specific reference values for several clinical parameters derived from a diverse European population of healthy children. These papers are further supplemented by online materials that present additional percentiles based on the total sample, including, for example, obese children and results of sensitivity analyses. In addition, public health professionals often need orientation values for parameters in order to describe and compare the health status and health behaviour of population groups. Similarly, parents and teachers may be concerned whether children in their care are developing well. In these cases, key factors impacting on the health status of children, such as nutrition and physical activity, will also be of interest.

Part 2 of this supplement describes the distribution of behaviours and characteristics related to energy balance in order to guide public health professionals and those caring for individual children to assess children's health-related behaviours. At the same time, these papers throw a spotlight on the current prevalence of several health-related factors of young children in
Europe, wherein we have to keep in mind that observed ranges-for example, of physical activity levels-do not necessarily reflect optimal levels.

Ideally, reference values should be quantitatively linked to subsequent health outcomes. This would require longitudinal observation over several decades. Unfortunately, such data are not available. Thus, the calculation of cutoff values based on the distribution in healthy children remains the most desirable approach at hand. Our cross-sectional data allow the analysis of the distribution of several markers that have proved to be associated with disease risk in adults and to describe the age- and sex-specific range of these markers in healthy children. It is a particular strength of our data set that it draws on a large population-based sample of (largely but not exclusively) Caucasian children from various regions-from Spain in the West to Estonia in the East, and from Sweden in the North to Cyprus in the South of Europe. Hence our data may serve as a reference for other Caucasian populations of children.

The IDEFICS survey was not intended to provide a nationally representative sample for the participating countries-such an endeavour would have far exceeded the available resources. Nevertheless, given the population-based approach and in contrast to the clinical populations often used for the calculation of reference values, our study sample should be considered as an unselected population. The IDEFICS communities were chosen because their socio-demographic profiles resembled those of the surrounding regions. Similar to other cross-sectional studies, a non-response bias caused by over-representation of certain population subgroups may have led to biased prevalence estimates, where the parameter of interest is associated with a factor affecting the willingness to participate in the study. Social position is, of course, a key factor associated both with participation and many health outcomes.

Does this mean that cross-sectional data are inappropriate for deriving valid reference values for clinical parameters? Not necessarily: there is no reason to believe that parameters of interest such as C-reactive protein, lipids or insulin are directly linked to social position. Such an association may well be mediated through weight status. Since our calculation of reference values is typically restricted to normal-weight children, we can assume that the presumed selection effects should not impair the validity of the derived percentile curves. Furthermore, for each parameter of interest we defined the analysis group by excluding comorbid conditions that have a pathological impact upon it. Therefore, we would argue that our sample represents an optimal population to generate reference values as it is likely to represent the healthier segment of the population. In this regard, it resembles the strategy that the $\mathrm{WHO}$ has followed-that is, to generate growth standards by defining children with optimal health. $^{2}$

All of the papers in this supplement apply the Generalised Additive Models for Location Shape and Scale, a recently developed statistical approach for the calculation of reference values that extends the well-established LMS method for deriving reference curves ( $\mathrm{L}=$ skewness, $\mathrm{M}=$ median, $\mathrm{S}=$ coefficient of variation). ${ }^{3}$ The application of this method to specific variables and markers is briefly described in each paper, along with the fitted distribution for calculating the percentiles of the variables or 
blood markers of interest. Sensitivity analyses were performed to investigate the robustness of the obtained percentiles with respect to the fitted distribution and varying definitions of the analysis group. Flow charts summarise the exclusion criteria and indicate the number of subjects excluded as well as those remaining for the main analysis. Since weight status is an important determinant for many of the parameters analysed here, we uniformly define analysis groups in line with the criteria by Cole and Lobstein published in 2012 for underweight, normal weight, overweight and obesity. ${ }^{4}$

To promote comparability, all our authors present the same percentiles (viz. 1st, 3rd, 10th, 25th, 50th, 75th, 90th, 97th and 99th). Some papers also present additional percentiles to facilitate comparisons with previously published data. For markers that lack clinical relevance we omitted the most extreme cutoffs. Similar abbreviations, symbols and colours are used in all papers to depict the various percentiles, to enable the reader to switch easily between papers. Although the number of subjects is given for each subgroup, it should be kept in mind that the reference curves are fitted on the assumption of an underlying continuous functional relationship, for example, between age and the corresponding percentiles.

\section{CONFLICT OF INTEREST}

The authors declare no conflict of interest.

\section{ACKNOWLEDGEMENTS}

We would like to express our gratitude to all the children and families who participated in the IDEFICS study and enabled this work. We hope that their contributions and the reference values presented here will assist the daily practice of paediatricians and other health professionals and contribute to many children's future well-being. We are grateful to Professor Tim Cole for fruitful discussion and many constructive suggestions, which have helped to improve this supplement.
We very much appreciate the contribution of the authors of this supplement and the enormous efforts of the whole IDEFICS consortium in creating this unique database. We would also like to record our thanks to the Volkswagen Foundation for its financial support in the production of this supplement.

W Ahrens ${ }^{1,2}$, LA Moreno ${ }^{3}$ and I Pigeot ${ }^{1,2}$

${ }^{1}$ Leibniz Institute for Prevention Research and Epidemiology-BIPS,

Bremen, Germany;

${ }^{2}$ Institute of Statistics, Faculty of Mathematics and Computer Science, Bremen University, Bremen, Germany and

${ }^{3}$ GENUD (Growth, Exercise, Nutrition and Development) Research Group, University of Zaragoza, Zaragoza, Spain

E-mail: ahrens@bips.uni-bremen.de

\section{REFERENCES}

1 Ahrens W, Bammann K, Siani A, Buchecker K, De Henauw S, lacoviello L et al.on behalf of the IDEFICS consortium. The IDEFICS cohort: design, characteristics and participation in the baseline survey. Int J Obes 2011; 35: S3-S15.

2 WHO Multicentre Growth Reference Study Group. WHO Child Growth Standards: Length/height-for-age, Weight-for-age, Weight-for-length, Weight-for-height and Body Mass Index-for-age: Methods and Development. WHO: Geneva, Switzerland, 2006.

3 Rigby RA, Stasinopoulos DM. Generalized additive models for location, scale and shape. J R Stat Soc Ser C (Appl Stat) 2005; 54: 507-554.

4 Cole TJ, Lobstein T. Extended international (IOTF) body mass index cut-offs for thinness, overweight and obesity. Pediatr Obes 2012; 7: 284-294.

(c) (1) $(-)$ This work is licensed under a Creative Commons Attributioncc) NonCommercial-NoDerivs 4.0 International License. The images or other third party material in this article are included in the article's Creative Commons license, unless indicated otherwise in the credit line; if the material is not included under the Creative Commons license, users will need to obtain permission from the license holder to reproduce the material. To view a copy of this license, visit http:// creativecommons.org/licenses/by-nc-nd/4.0/ 